\title{
Die Bedeutung der Röntgenstrahlen im Kampfe gegen die Tuberkulose.
}

Von

Dr. med. Gustar Loose in Bremen.

In den Röntgenstrahlen haben wir ein Mittel gefunden, das wie kein anderes und in idealster Weise imstande ist, jede pathologische Zelle, resp. jede pathologische Anhäufung von Zellen restlos zu vernichten und zu zerstören. Es. kommt einzig und allein darauf an, die jeweilig wirksamste Wellenlänge in genügender Dosis und obne Schädigung für Haut und Gesamtorganismus in dem Krankheitsherde zur Entfaltung zu bringen. Von dem Tage an, an dem die Fortschritte der Technik uns hierzu befähigen, wird es eine Revolution in der Medizin geben, wie kaum je zuvor, da ja ausgedehnte Gebiete und zahlreiche Leiden auf pathologischem Zellwachstum beruhen. Ein lehrreiches Beispiel für den Grad der Umwälzung bietet uns die Röntgenbebandlung gutartiger gynäkologischer Leiden (Myome, Menorrhagien usw.). Wenn wir uns einmal an die Kämpfe in den Jahren zurückerinnern, in denen die Röntgenstrahlen als scharfe Konkurrenten der operativen Behandlung auftraten, und ferner einmal die Jetztzeit zum Vergleich heranziehen, so sehen wir, dass der Sieg des Röntgenlichtes ein so vollendeter ist, dass wir die Akten über dieses Thema als geschlossen urd erledigt betrachten können. Genau so wird es allen andern gutartigen Geschwälsten ergehen, die röntgenologisch in dieselbe Gruppe gehören, z. B. der Struma (gleichgültig welcher Ätiologie), der Prostatabypertrophie usw. Genau'so wird es dem gesamten Karzinom ergehen, da die glänzenden technischen Fortschritte der letzten Jahre uns zu den besten Hoffnungen berechtigen. Wie stark bereits die Konkurrenz der Röntgenstrahlen auch hier in Erscheinung tritt, beweisen die letzten diesbezüglichen Publikationen (s. Krönig und Friedrich; Seitz und Winz; Verfasser u. a.).

Welchen Einfluss üben nun die Röntgenstrahlen auf die Tuberkulose aus, die ja auch, wie schon ihr Name sagt, auf Geschwulstbildung beruht? 
Grosse Gebiete tuberkulöser Erkrankungen sind bereits von den Röntgenstrahlen erobert worden; so z. B. die überaus häufige Lymphdrüsentuberkulose, einerlei ob sie am Halse, in den Leisten, im Mediastinum, im Abdomen oder sonstwo sich lokalisiert. Wenn ich von den hiesigen Verhältnissen auf die Allgemeinheit schliessen darf, so scheint mir die Röntgenbehandlung dieses Leidens so sehr schon Allgemeingut der Ärzteschaft geworden zu sein, dass die bisherige operative Behandlung als veraltet und überholt sich auf dem Aussterbeetat befinden. Ähnliche Erfolge lassen sich bei mancherlei anderen tuberkulösen Herderkrankungen erzielen, z. B. bei kalten Abszessen, Fisteln usw.

Das Hauptinteresse erfordert jedoch, wie überhaupt bei der Tuberkulose, die Frage: welchen Einfluss üben die Röntgenstrahlen auf die verbreitetste und gefährlichste Form, die Lungentuberkulose aus? Es sind bekanntlich bereits vor mehreren Jahren von Freiburg aus Erfolge bekannt gegeben, die so gut waren, dass die Röntgenbehandlung der Lungentuberkulose dort mit in den ständigen Heilplan der Klinik, resp. des Sanatoriums aufgenommen wurde. Hierdurch angeregt habe ich, zum Teil in Verbindung mit einem hiesigen tuberkuloseerfahrenen Kollegen, eine kleine, absichtlich stark beschränkte Anzahl sicherer Fälle von Lungentuberkulose in Röntgenbehandlung genommen. Der Erfolg war, dass bei fast allen leichteren, älteren, aber nie ganz verheilten Formen der Einfluss so weit ging, dass sie als ausgeheilt bezeichnet werden konnten. Bei frischeren Erkrankungen traten in der überwiegenden Mehrzahl wesentliche, zum Teil überraschende Besserungen ein: Es decken sich also meine Erfahrungen mit den Freiburgern. Wenn es natürlich auch noch zu früh ist, sich ein abschliessendes Urteil zu bilden, so scheinen doch die Röntgenstrahlen sich zu einer Waffe gegen die Lungentuberkulose zu entwickeln, die mit den Fortschritten der Technik eine immer wirksamere werden dürfte.

Die Hauptbedeutung der Röntgenstrahlen im Káampfe.gegen die Lungentuberkulose liegt jedoch auf einem ganz anderen als dem eben geschilderten Gebiete, nämlich dem der Prophylaxe, resp. Immunisierung.

Es sei mir gestattet, an dieser Stelle kurz einige allgemeine Be. merkungen einzuflechten. Wer, wie Verfasser, Gelegenheit gehabt hat, viele Jahre hindurch ein grosses, reichhaltiges Krankenmaterial mit zu untersuchen, der muss sich immer wieder darüber wundern, welch riesige Verbreitung einerseits die Lungentuberkulose gewonnen hat, andererseits, wie machtlos wir im grossen und ganzen ihr gegenüberstehen. Es liegt mir nichts ferner, als etwa unsere Heilstättenbestrebungen bemängeln zu wollen. Aber die Zahl derer, die in einer Heilstätte aufgenommen werden und aufgenommen werden können, stellt doch immerhin nur einen recht bescheidenen Prozentsatz dar. Ziehen wir ferner in Erwägung, dass von sämtlichen Heilstättenpatienten doch nur wiederum ein gewisser Teil wirklich daternd geheilt wird, so werden die Resultate noch betrübender. Der Krieg hat uns so recht und mit furchtbarster. Deutlichkeit gezeigt, dass jeder, der einmal von der Lungentuberkulose befallen gewesen ist, 
sein Leben lang unter einem Damoklesschwerte wandelt. Dje Renten, die wir nach dem Kriege werden bezahlen müssen, werden in erschreckender Weise lehren, wie zahllos die Fälle sind, in denen Tuberkulöse, die sich dauernd geheilt glaubten und jahrelang, bewusst oder unbewusst, beschwerdefrei gewesen waren, durch die Ungunst der Verhältnisse plötzlich und rapide wieder Aktivtuberkulöse wurden. Ferner wird, glaube ich, jeder von uns in seiner Praxis oder in seinem Bekanntenkreise jene traurigen Fälle erlebt haben, wo trotz der glänzendsten äusseren Verhältnisse und trotz aller uns zu Gebote stehenden Mittel blühende Menschenleben und anscheinend leicht Erkrankte einer gallopierenden Schwindsucht erlagen. Wir müssen ruhig zugeben, dass unsere ganze Tuberkulosetherapie ein klägliches Fiasko bedeutet, und dass uns eine wirkliche vernichtende Waffe zur Zeit noch fehlt.

Der Grund nun, warum wir in diesem Kampfe nicht recht weiter kommen, liegt darin, dass Zeit und Ort des Angriffs falsch gewählt sind; mit andern Worten, dass wir immer erst warten, bis dieser gemeingefährliche Gegner sich fest im Lande eingenistet hat, so fest, dass es mit unseren heutigen Mitteln nur in wenigen Fällen gelingt, ihn dauernd wieder hinauszujagen. Diese Erkenntnis führt uns ganz von selbst $\mathrm{zu}$ der Frage hinüber: wie und wann entsteht die Tuberkulose? Es kann nicht die Aufgabe dieser Zeilen sein, von der schwierigen, verwickelten und heiss umstrittenen Sachlage eine erschöpfende Übersicht zu bieten, sondern ich beschränke mich auf eine kurze Skizzierung, der ich einige persönliche Bemerkungen beifügè. In der Frage des Modus der Tuberkuloseinfektion stehen sich im grossen und ganzen immer noch zwei Parteien gegenüber. Die eine vertritt die Ansicht $\mathrm{Kochs}$, dass der Respirationstraktus die Haupteingangspforte bildet. Die andere folgt den Anschauungen Behring-Römers, die dem Digestionstraktus die Hauptrolle zuerteilen. Mag dem nun sein, wie es will; für die heutigen Betrachtungen möge der Kampf einmal dahin entschieden sein, dass, wie übrigens durchaus wahrscheinlich, beiden Wegen die gleiche Bedeutung beizumessen ist. Wann entseht nun die Infektion? Wie weit auch hier wieder die Ansichten auseinander gehen mögen, darüber herrscht doch im allgemeinen Einigkeit, dass nämlich wohl jeder Mensch einmal in seinem Leben die Tuberkaloseinfektion durchmacht, genau wie jedes Kind einmal von den Masern befallen wird. Für die heutigen Betrachtungen kann wiederum der Widerstreit der Meinungen gleichgültig sein, ob die Infektion eine schwere Gefahr für den Organismus darstellt, also möglichst verhütet werden soll, oder ob wir genau umgekehrt in ihr ein gütiges Walten der Natur zu erblicken haben, die durch die Anregung der Gegengiftwirkung bestrebt sein will, die Gefahr der Infektion im späteren Leben herabzusetzen. Fassen wir nun die Tuberkulose als eine Infektionskrankheit wie jede andere auf, dann wird auch ihr Auftreten in der Hauptsache im jugendlichen Alter zu suchen sein. Befällt sie einen jugendlichen kräftigen Körper, so wird dieser leicht imstande sein, durch den Überschuss seiner Kraft den Feind zu vernichten oder durch die durch nichts gehemmte Gegengiftbildung den Angriff ab- 
zuschlagen. Befällt sie ein schwächliches Kind, so wird die Abwebr ihm schwer fallen und vielleicht für das spätere Leben eine leicht verwundbare Lücke offen lassen. Befällt sie einen kranken oder erblich stark belasteten Organismus, so wird der Kampf ein so ungleicher und aussichtsloser sein, dass ihm über kurz oder lang das Leben erliegt.

Wie ausgedehnt bereits im Kindesalter die Tuberkulose ist, lehren zahlreiche Statistiken; als letzte, soweit ich die Literatur übersehe, die. von Späth in der Münch. med. Wochenschr. 1918, Nr. 13, veröffentlichten Zahlen, nach denen von 153 Schulkindern auf Grund der Pirquetschen Kutanreaktion nur 12,3\% biologisch und klinisch frei von Tuberkulose waren. Diese betrübenden Verhältnisse müssen immer wieder zu der Erkenntnis führen, dass einzig und allein der Staat mit Hilfe des Gesetzes imstande sein wird, eine programmmässige Tuberkulosebekämpfung in die Wege zu leiten. Der Ruf nach Zwangsuntersuchungen und Zwangsaufsicht unserer Schulkinder ist ja nicht neu. Er ist immer wieder erhoben worden, hat aber zu irgendwelchen Erfolgen nicht geführt. Warum die massgebenden Behörden diesem Verlangen bisher ablehnend gegenüberstanden, entzieht sich meiner Kenntnis. Aber es kann und darf nicht anders kommen, als dass "unsere gesamte Jugend, genau wie den Pocken, so auch der Tuberkulose gegenüber einer scharfen; durch das Gesetz genau festgelegten Aufsicht unterstellt wird.

Warum lasse ich nun gerade und gerade jetzt diesen Ruf von neuem ertönen, obgleich er bisher resultatlos verhallt ist? Weil ich der Überzeugung bin, dieser hochbedeutsamen Frage durch zwei neue Gesichtspunkte ein ganz anderes, erfolgverheissendes Aussehen verleihen zu können. Der erste betrifft die diagnostische, der zweite die therapeutische Seite.

$\mathrm{Zu}$ 1: Wie auf dem gesamten Gebiete der Medizin, so werden wir auch der Lugentuberkulose gegenüber desto bessere Heilungsaussichten besitzen, je eher das Leiden erkannt wird. Ich schliesse mich auf Grund einer jahrelangen, an reichhaltigem Material gewonnenen Erfahrung durchaus jenen, sich immer lauter erhebenden Stimmen an, die vor einer Überwertung der alten Untersuchungsmethoden warnen (siebe z. B. Hein ecke, Münch. med. Wochenschr. 1918, 15-16). Ich behaupte hier, dass wir zur Entscheidung der Frage, ob in der Lunge überhaupt organische Veränderungen vorliegen oder nicht, in den Röntgenstrahlen ein Mittel besitzen, das der gesamten Auskultation. und Perkussion konkurrenzlos überlegen ist. Ich bin mir sehr wohl bewusst, auf welch ungeheuren Widerspruch diese Behauptung, die wohl zum ersten Male so offen ausgesprochen wird, bei der älteren Schule stossen wird; aber das macht nichts; je härter der Kampf, desto schöner der Sieg, und ich hoffe, dass unsere nächsten Kongresse uns recht bald Gelegenheit geben werden, ausgiebig über diese Frage zu debattieren oder aber, wenn es sein soll, auch die Möglichkeit bieten, den praktischen Beweis zu erbringen, wer recht oder unrecht hat. Allerdings bedarf es einer exakten röntgenologischen Technik, auf die ich mit einigen Worten eingehe. Erkennen wir bei einer Lungenuntersuchung mit gut adap- 
tiertem Auge schon auf dem Leuchtschirm tuberkulöse Veränderungen, so handelt es sich stets um gröbere, meist ausgedehntere Prozesse. Lässt sich nichts Sicheres nachweisen, pflege ich in meinem Protokoll zu bemerken: ohne gröberen pathologischen Befund. Handelt es sich um einen feineren Prozess oder um Tuberkuloseverdacht, würde es einen schweren Kunstfehler bedeuten, mit der Ieuchtschirmuntersuchung die Röntgenuntersuchung abzuschliessen. In all diesen Fällen muss unbedingt die Röntgenplatte herangezogen werden, weil sie ein viel feineres Reagens als die Netzhaut unserer Augen ist, und man muss sich häufig wundern, wie deutlich auf der Platte Spitzenaffektionen zum Ausdruck kommen, die sich der Leuchtschirmuntersuchung völlig entzogen. Des weiteren ist dringend davor $\mathrm{zu}$ warnen, etwa aus dem Bestreben der Abkürzung der Exposition, sich eines Verstärkungsschirmes, gleichgültig welcher Herkunft, zu bedienen. Drittens kann nie genug empfohlen werden, Röntgenstereogramme zu machen, wobei die Abkürzung der Exposition dem Geschmacke des Einzelnen überlassen werden kann. Wer einigermassen Übung in der Deutung ron Röntgenstereogrammen besitzt, wird sich beim Vergleich der einfachen und der Doppelplatte wundern, wieviel mehr diagnostische Feinheiten sich aus der letzteren herauslesen lassen, und wird von Tag zu Tag mehr seine Freude an der Sicherheit der Diagnose erleben. In vielen Fällen, besonders leichteren, erhält man einen derartigen Einblick in die wahren Verhältnisse, dass man nur nach dem Röntgenbilde, ohne überhaupt zu auskultieren und zu perkutieren, den Auskultations- und Perkussionsbefund diktieren kann. Ich habe mir gelegentlich durch Stichproben das Vergnügen geleistet, den ganz unabhängig voneinander aufgenommenen und auf diese Weise gewonnenen Befund mit dem der betreffenden klinischen Abteilung zu vergleichen. Sie stimmten vielfach derartig überein, dass der eine von dem andern abgeschrieben sein konnte.

Worauf begründet sich nun die zunehmende Misskreditierung der Auskultation und Perkussion? Sicherlich nicht auf den Mangel an technischer Gewandtheit oder Mangel an Übung, sondern auf der Erkenntnis, dass die physikalischen Verhältnisse in zahlreichen Fällen so ungünstig liegen, dass sie bei der Auskultation und Perkussion $\mathrm{zu}$ unsicheren oder falschen Resultaten führen müssen. Als ungünstiges Moment für die Perkussion erwähne ich z. B. starre Thoraxformen, die resonanzbodenartig wirken; muskulöse und adipöse $\mathrm{Pa}$ tienten oder dergleichen; für die Auskultation feinere, tiefgelegene Veränderungen, die sich ihr völlig entziehen. Gerade für die Lungentuberkulose haben aber die tiefgelegenen, am Hilus lokalisierten Herde eine ausserordentlich grosse Bedeutung, da sie nach meiner Überzeugung in allen vom Respirationstraktus ausgehenden Fällen den Anfang bedeuten. Warum nun in dem einen Falle die Tuberkulose vom Hilus aus dem Bronchialbaum folgt und sich fächerartig im Lungenfelde ausdehnt, in dem andern Falle sich in begrenzten Gebieten, z. B. den Spitzen, einnistet, ist ungeklärt. Vielleicht hängt es mit den sehr verschiedenen Thoraxformen zusammen. Immer aber ist, auch bei leichteren Spitzenerkrankungen, die Mitbeteiligung des Hilus an Drüseneinlagerungen und leichten Infiltrationen zu erkennen. 
Über den Prozentsatz der Hilus- und anderweitig lokalisierten Tuberkulose habe ich statistische Angaben in der Literatur nicht finden können. Nach meiner Erfahrung ist die erstere Form wesentlich häufiger als man im allgemeinen anzunehmen scheint.

Ähnlich liegen die Verhältnisse im Abdomen. Verkalkte Mesenterialdrüsen als Uberreste, aber auch als Beweis einer überstandenen Tuberkuloseinfektion sind als Nebenbefund bei den Sektionen überaus häufig und jedem Pathologen geläufig. Es liegt deshalb der Schluss nahe - und nach meiner Überzeugung ist es so - dass das lymphatische System, wenn nicht die direkte Eingangspforte, so doch den ersten grossen Schutzwall bildet, über den der Organismus gegen den Tuberkuloseangriff verfügt. Deshalb muss es unser Streben sein, nicht erst den Angriff abzuwarten, sondern möglichst früh unsere Schutzmassregeln derartig auszubauen und zu stärken, dass ein Durchbruch so leicht nicht möglich ist. Warum sollte der Tuberkuloseinfektionsmodus ein anderer sein als etwa bei der Wundinfektion? Nehmen wir eine harmlose Fingerwunde als Beispiel, so sehen wir regelmässig das lymphatische System als Filter und Damm automatisch in Funktion treten, sobald die lokalen Gegenmittel des Körpers nicht genügen. Wird es durchbrochen, kommt es zur Allgemeininfektion, die sich irgendwo im Körper lokalisieren kann.

Zu 2: Wenn wir auf Grund der oben geschilderten Verbältnisse die Annahme als richtig voraussetzen, dass jeder Mensch eine Tuberkuloseinfektion durchmacht, und ferner, dass die Infektionszeit hauptsächlich in der Jugend zu suchen ist, so müsste man auch annehmen, dass hierbei klinische Symptome sich geltend machen. Dies ist in der Tat der Fall, und das Krankheitsbild der kindlichen Tuberkuloseinfektion ist ein so gut umschriebenes, dass es so leicht nicht übersehen werden kann. Meist handelt es sich um Kinder von eben vor- oder gerade schulpflichtigem Alter, die bisher eine völlige normale geistige und körperliche Entwickelung durchgemacht hatten und die nun ohne irgendwelche äussere Veranlassung zu kränkeln anfangen, $d$. h. in ibren geistigen und körperlichen Fähigkeiten nachlassen, leicht ermüden, appetitlos werden, über Kopf- und Leibschmerzen klagen, gelegentliche abendliche Temperatursteigerungen aufweisen, kurz den Eindruck erwecken, als ob ihr junger Körper gegen irgend einen versteckten und verkappten Feind ankämpft. Alle diese Kinder, behaupte ich, befinden sich im Stadium der Tuberkuloseinfektion, selbstverständlich vorausgesetzt, dass andere Ursachen (Darmparasiten, Blutkrankheiten oder dergl.) ausgeschlossen sind.

Wenn ich des weiteren auf die eingangs dieser Betrachtungen aufgestellte Ansicht verweise, dass wir jede auf Geschwulst- oder Geschwülstchenbildung beruhende Erkrankung durch Röntgenstrahlen beeinflussen und heilen können, sobald uns die spezifisch wirksame Wellenlänge zur Verfügung steht, so sollte man erwarten, dass auch bei der jugendlichen Tuberkuloseinfektion ein günstiger Einfluss vorhanden sein muss. Ich habe nun schon seit mehreren Jahren mir eine absichtlich beschränkte Anzahl von Kindern, die mir den oben beschriebenen Symptomenkomplex aufzuweisen schienen, in Röntgenbehandlung, auf deren genauere Technik ich hier nicht eingehen 
kann, genommen, mit dem Erfolge, dass fast ausnahmslos sich eine wesentliche, in vielen Fällen geradezu verblüffende Besserung geltend machte. Man könnte den Umschwung vergleichen mit dem Aufblühen von Blumen im Sonnenlichte, die vorher im Schatten gedarbt hatten.

Als erstes Anzeichen des Röntgeneinflusses macht sich nach meiner Erfahrung die Zunahme des Appetites bemerkbar, eine sehr angenehme Zugabe, da eine möglichst reichhaltige Ernährung immer noch einer unserer besten Bundesgenossen ist. Die Esslust der Kinder war in einer Reihe. von Fällen derartig in Zunahme, dass es, wie die Eltern mir fast vorwurfsvoll berichteten, schwerfiel, sie, zumal unter den Kriegsverhältnissen, satt zu bekommen. Mit zunehmender Röntgenwirkung schwinden dann nach und nach alle übrigen Krankheitserscheinungen, so dass sowohl geistig wie körperlich die Kinder wieder einen völlig gesunden Eindruck machen. Kurz vor Niederschrift dieser Zeilen habe ich mich bemüht, von der ersten Serie, die schon längere Zeit ausser Behandlung ist, Nachricht zu erhalten. Soweit ich Antworten bekam, lauteten sie durchaus günstig; der Gesundheitszustand̉ liess nichts zu wünschen übrig.

Als Probe aufs Exempel veröffentliche ich einen einzigen Fall, dessen Anamnese und Verlauf ich kurz wiedergebe. Es handelt sich um meine eigene älteste Tochter, Minnie L., geb. Okt. 1911; normales kräftiges Kind mit normaler Entwickelung; abgesehen von einzelnen Lungentuberkulosefällen in der entfernteren Verwandtschaft mütterlicherseits keine Tuberkelhereditas. Seit etwa einem Jahr Nachlassen in der Gesundheit durch blasses Aussehen, leichte Ermüdung, Klagen über Kopf- und Leibschmerzen usw. Nach Beendigung einer Kur gegen Vermes filiformes, die als Ursache in Betracht kommen konnten, keine nennenswerte Besserung, so dass ich mich zur Diagnose: $\mathrm{Tu}$ berkuloseinfektion entschloss und Pat. selbst bestrahlte. Schon nach den ersten Dosen hervorragende Besserung, zuerst kenntlich an einer enormen Appetitsteigerung, so dass es augenblicklich in der Tat schwer ist, das Nahrungsaufnahmebedürfnis immer zu stillen. Im Laufe der nächsten Wochen verschwinden so gut wie alle Beschwerden, so dass das Kind jetzt geistig und körperlich wieder einen frischfröhlichen Eindruck macht, und auf diese Weise wohl vorbereitet seine Schuljahre angetreten hat.

Dieser Erfolg hat mich in meiner Überzengung so gekräftigt, dass ich auch meine übrigen Kinder, sobald sie sich in den Jahren befinden, prophylaktisch mit Röntgenstrahlen behandeln werde.

Diese Bemerkung führt uns von selbst zu der Frage hinüber: wann ist die günstigste Zeit für eine derartige Behandlung?

Während bei der Röntgendiagnostik Untersuchungen kleiner und kleinster Kinder keine Schwierigkeiten mehr bereiten, da man, falls dieselben unruhig sind, in der Exposition bis auf Bruchteile von Sekunden heruntergehen kann, so ist dies in der Therapie bisher nicht möglich, m. E. auch wegen Gefährdung der Dosierungssicherheit nicht zu empfehlen. Es ist daher notwendig, dass die Kinder eine Zeitlang, durchschnittlich etwa 10-15 Minuten, ruhig liegen. Dies ist nur zu erreichen, wenn die kleinen Patienten bereits über einen gewissen 
Grad eigener Vernunft verfügen und dem Erziehungsgehorsam zugängig sind. Auf der andern Seite lehrt die Statistik, dass unter Schulkindern bereits zahlreiche Tuberkulöse sind, die Schule also, wie bei allen anderrn Infektionskrankheiten, so auch in unserem Falle als eine nicht ausser Acht zu lassende Infektionsquelle zu betrachten ist. Es muss dementsprechend vor Eintritt in die Schule die Röntgenbehandlung beendet sein. Mir scheint aus den angeführten Gründen das 5. Lebensjahr, falls nicht besondere Verhältnisse vorliegen, der richtigste Zeitpunkt zu sein.

Von manchem Leser dieser Zeilen wird sicher die Frage aufgeworfen werden: ist es nicht gefährlich oder gewagt, bei kleinen Kindern Röntgenbestrahlungen vorzunehmen? Können nicht ferner neben der beabsichtigten Wirkung. Schädigungen auftreten, die die Vorteile wieder wettmachen oder gar übertrumpfen? Beide Fragen sind mit einem völlig sicheren, glatten Nein zu beantworten!. Denn einmal hat die Sicherheit der Dosierungstechnik derartige Fortschritte gemacht, dass sie in der Hand des Fachmannes so leicht nicht versagen wird; vor laienhafter Röntgentherapie kann allerdings nicht scharf genug gewarnt werden. Auf der andern Seite ist die erforderliche Dosis eine so geringe, dass die Schädigung normaler kindlicher Zellen ausgeschlossen ist. Das ist doch gerade das Ideale der Röntgenstrahlen, dass sie spezifisch auf die kranke Zelle einwirken, während" sie die normale völlig intakt lassen. Dieselbe Frage ist schon vor vielen Jahren aufgeworfen, und es haben Versuche an jungen Tieren ergeben, dass an normalen Zellen Schädigungen, z. B. des Wachstums, erst durch derartig bohe Dosen zu erreichen sind, wie sie am Menschen überhaupt nicht in Frage kommen. Im übrigen lassen sich besonders röntgenempfindliche Organe, wie Ovarien, Testikel oder Milz bei der Bestrahlung ja leicht abdecken und so sicher schützen. Das einzige, was bei empfindlichen Kindern mit in den Kauf genommen werden muss, sind die Erscheinungen eines leichten Röntgenkaters, bestehend in leichtem Übelsein, Kopfschmerzen usw., die jedoch bereits am nächsten Tage ausnahmslos wieder verschwunden sind.

Wie ist nun der therapeutische Einfluss der Röntgenstrahlen zu erklären? Sicherlich nicht etwa durch die Abtötung eingedrungener Tuberkelbazillen. Denn die schon vor Jahren angestellten Versuche haben ergeben, dass die Abtötung von Kulturen im Reagenzglas erst durch so hohe Dosen möglich ist, dass sie beim Menschen ohne schwere Schädigung nicht angewendet werden dürfen. Allerdings stammen die Versuche aus einer Zeit, in der man die moderne Tiefentherapie noch nicht kannte, und ich habe in der Literatur keinen Hinweis gefunden, ob sie mit moderner Technik nachgeprüft worden sind. Trotzdem bleibt der direkte Einfluss unwahrscheinlich. Zwwanglos wird die Erklärung, wenn wir uns den Inhalt der ersten Zeilen ins Gedächtnis zurückrufen. 'Es würde demnach der Vorgang so sein, dass die durch den Bazillus oder seine Giftstoffe zu pathologischem Wachstum (Riesenwuchs) veranlasste Zelle zurückgebildet oder zerstört wird. In andern Worten: es würde durch den Röntgeneinfluss dem Bazillus der Nährboden entzogen, er selbst durch Aushungerung vernichtet und seine Vermehrung verhindert. Die Behring-Rö mer sche Theorie 
würde dieser Ansicht sich so anpassen, dass die durch die eingedrungenen Bazillen oder ihre Gifte angeregte Antitoxinbildung durch den Röntgeneinfluss verstärkt wird und weiterschreitet, die anfangs noch geringe Zahl der Feinde tötet und eine so ungeheure Masse von Gegengiften als Reserve im Organismus aufstapelt, dass zukünftige Angriffe mit Leichtigkeit abgeschlagen werden können.

Fasse ich nun zum Schlusse den Inhalt dieser Zeilen kurz zusammen, so möchte ich folgende Leitsätze aufstellen:

1. Unser Kampf gegen die Lungentuberkulose ist bisher deshalb von so geringem Erfolge gewesen, weil uns einmal ein radikales Heilmittel immer noch fohlt, andererseits der Kampf gegen diese verbreitetste Seuche viel zu spät begonnen wird.

2. Da jeder Mensch einmal eine Tuberkuloseinfektion durchmacht, und zwar in der Jugend, so muss unser Kampf auch in der Jugend beginnen.

3. Ein durchschlagender Erfolg ist nur durch staatliche Organisation und gesetzliche $Z$ wangsmassregeln zu erwarten.

4. Diese alte Forderung, die bisher stets vergeblich erhoben wurde, erhält erneute und wesentlich erhöhte Bedeutung durch zwei Errungenschaften der Neuzeit.

5. Denn in der Untersuchùng mit Röntgenstrahlen besitzen wir ein Mittel, das zur Entscheidung der Frage, ob bereits tuberkulöse Veränderungen in der Lunge vorhanden sind oder nicht, den alten Untersuchungsmethoden überlegen und in allen 2weifelhaften Fällen der ausschlaggebende Faktor ist.

6. Durch die Fortschritte der Technik scheinen sich die Röntgenstrahlen als direktes Heilmittel gegen die Lungentuberkulose zu einer wirksamen Waffe zu entwickeln.

7. Ihre Hauptbedeutung liegt auf dem Gebiete der jugendlichen Prophylaxe oder Immunisierung und beruht auf der Tatsache, dass einerseits die Tuberkulose in die Gruppe der Geschwulstkrankheiten gehört, andererseits wir in den Röntgenstrahlen ein Mittel besitzen, das in Kampfe gegen jede Geschwulstbildung jetzt schon als scharfer Konkurrent gegen die bisherige Bebandlung auftritt und in Zukunft ihr konkurrenzlos überlegen ,werden wird.

Mancher Leser dieser Zeilen wird bedenklich sein Haupt schütteln und denken: Phantast, Idealist, blühender Optimist oder dergleichen. Er möge seinen Gefühlen keinen Zwang antun. Mir haben nun einmal die Nornen den Optimismus in die Wiege gelegt und ich freue mich dessen. Denn noch niemals in der Welt hat Duckmäuserei oder Pessimismus einer Sache zum Siege verholfen und ohne Optimismus kein Fortschritt. „Das alte stürzt, es ändert sich die Zeit, und neues Leben blüht aus den Ruinen." Vielleicht ist der Tag nicht fern, an dem die gesamte deutsche Jugend vor ihrem Eintritt in die Schule und nach gesetzlicher Vorschrift wird Rechenschaft ablegen müssen über die Frage: in welchem Verhältnis stehst Du zur Tuberkulose? Vielleicht werden einmal die Röntgenstrahlen berufen sein, einen Jahrgang nach dem andern zu untersuchen und die Frage zu ent- 
scheiden, ob tuberkulosegesund, ob tuberkuloseverdächtig oder tuberkulosekrank. Vielleicht wird der Siegeslauf des Röntgenlichtes einmal dahin führen, im Bunde mit den übrigen Mitteln und Methoden die gesunden $\mathrm{zu}$ immunisieren, die verdächtigen $\mathrm{zu}$ schützen und die kranken zu bessern oder zu heilen. Dann kann eine neue Blätezeit in militärischer, sanitärer und sozialer Hinsicht nicht ausbleiben. Sollten die vorliegenden Betrachtungen dazu beitragen, ist ihr Zweck reichlich erfüllt.

Auch diesmal spreche ich die Bitte aus, mir Anfragen über technische Einzelheiten zu ersparen. Wir haben nun eben leider kein direktes Mittel, das eine leichte, sichere Verständigung ermöglicht, und die indirekten sind so verworren und werden so verschieden gehandhabt, dass sie die Gefahr in sich bergen, durch Missverständnisse Schädigungen hervorzurufen, die dem Ganzen ausserordentlich schaden können. Deshalb bitte ich auch diesmal wieder, sich bis zum nächsten Röntgenkongress zu gedulden. 